\title{
Impact of Hardware Impairments with Imperfect Channel Estimation for Cache-Enabled UAV Relaying Networks
}

\author{
Dan Deng, ${ }^{1}$ Yanyi Rao $\mathbb{D}^{2}$, and Fusheng Zhu $\mathbb{B D}^{3}$ \\ ${ }^{1}$ School of Information Engineering, Guangzhou Panyu Polytechnic, Guangzhou 511483, China \\ ${ }^{2}$ School of Computer Science and Cyber Engineering, Guangzhou University, Guangzhou 510006, China \\ ${ }^{3}$ Guangdong New Generation Communication and Network Innovative Institute (GDCNi), Guangzhou, China \\ Correspondence should be addressed to Yanyi Rao; raoyy@gzhu.edu.cn and Fusheng Zhu; zhufushengcom@126.com
}

Received 27 April 2020; Revised 10 June 2020; Accepted 26 June 2020; Published 10 August 2020

Academic Editor: Lisheng Fan

Copyright (c) 2020 Dan Deng et al. This is an open access article distributed under the Creative Commons Attribution License, which permits unrestricted use, distribution, and reproduction in any medium, provided the original work is properly cited.

The effects of hardware impairments with imperfect channel estimation for cache-enabled UAV networks are studied in this paper. The effects of the setup parameters, such as the number of cached or relaying nodes, the hardware impairment factor, the channel estimation error, and the transmission SNR, on the outage probability are present by deriving the exact closed-form expressions on outage probability. Also, the asymptotic results are present when the transmission SNR is large enough. From the asymptotic analysis, we can see that there is an error floor introduced by the hardware impairments and imperfect channel estimation. Specifically, the error floor is dependent on the channel estimation error factor, the hardware impairment distortion factor, and the QoS requirements. And the diversity order of the error floor for the cached/relaying links is equal to the number of cached/relaying UAV nodes.

\section{Introduction}

In recent years, wireless throughput is growing up exponentially $[1-3]$ and wireless communication network is facing more and more challenges introduced by wireless big data [4-7]. Cache technology, which can both improve the quality of service (QoS) of wireless link and relieve the pressure of wireless throughput, has demonstrated as a remarkable enabling solution for wireless networks [8-10]. By exploiting interference neutralization, the authors in [11] proposed an optimal content placement method to improve the system capacity. Furthermore, the maximum distance separable coding is adopted in wireless cache networks in [12], which can improve the system performance in terms of successful retrieval probability.

On the other hand, hardware impairments, which are introduced by phase noise of the RF components, show great effects on the system performance. Hardware impairments have been extensively studied and modeled as additive distortion noise [13] or nonlinear polynomial multiplicative fac- tor $[14,15]$. Considering amplify-and-forward (AF) relaying networks, the authors in [16] investigated the effects of hardware impairments on channel capacity by exact closed-form expression as well as the tight bounds, while Ref. [17] proved that larger hardware impairments can be used in massive MIMO due to the huge degrees of space freedom. By using iterative optimization algorithm, Ding et al. [18] proposed a solution to maximize the detection probability.

Meanwhile, due to the high mobility, there exists imperfect channel estimation since the linear estimation methods are applied on pilot signals [19]. Considering correlated Rayleigh fading with imperfect channel status information, Al-Hussaibi and Ali [20] derived expressions on the ergodic capacity with antenna selection. The authors in [21] analyzed the impact of imperfect CSI estimation for Alamouti-OSTBC Wireless Cooperative Networks in terms of symbol error probability.

However, the impacts of hardware impairments with imperfect channel estimation on relaying networks are still open questions. Considering unmanned aerial vehicle (UAV) 
networks, the UAV nodes work as a cache enable access point or an AF relaying node. To improve the QoS of the wireless channels, the best cached UAV nodes or AF relaying nodes are selected to help the message transmission from the macro BS to the user. The effects of the system parameters, such as the number of cached/relaying nodes, the hardware impairment factor, the channel estimation error, and the transmission SNR, on the outage probability are present by deriving the exact closed-form expressions on outage probability. Also, the asymptotic results are present when the transmission SNR is large enough. From the asymptotic analysis, we can see that there is an error floor introduced by the hardware impairments and imperfect channel estimation. Specifically, the error floor is dependent on the channel estimation error factor, the hardware impairment distortion factor, and the QoS requirements. And the diversity order of the error floor for the cached/ relaying links is equal to the number of cached/relaying UAV nodes.

The main contributions of this paper are as follows:

(i) Cache-enabled UAV relaying protocol is adopted to improve the QoS of the wireless links, where the UAV nodes work as a cache enable access point or an AF relaying node

(ii) We provide the deep insight on the effects of the system parameters, such as the number of cached/relaying nodes, the hardware impairment factor, the channel estimation error, and the transmission SNR, on the outage probability by deriving the exact closed-form expressions on outage probability

The organization of this paper is as follows. Section 2 presents the system model of cache-enabled amplify-andforward UAV networks, while the UAV selection algorithm and its performance analysis are given in Section 3. Furthermore, the asymptotical analysis is present in Section 4 with large transmission power. In Section 5, simulation results are present to validate the theoretical analysis. Finally, conclusion is given in Section 6.

\section{System Model}

The system model of cache-enabled amplify-and-forward unmanned aerial vehicle (UAV) networks is present in Figure 1, where there is one destination user, one macro base station (BS) and $N$ cache-enabled UAV relaying nodes. We assumed that all nodes are equipped with only one antenna. Furthermore, because of the shadow fading, there is no direct link from the macro BS to the destination user. In order to improve the QoS of the links, one of the UAV nodes is selected to assist the message transmission. Since the UAV nodes hold limited cache memory, the backhaul load from the macro BS to the UAV nodes can be relieved if the requested files from the user are hit in the UAV nodes. In this case, one of the UAV nodes is selected and directly transmits message to the user. On the other hand, if the requested file is not cached,

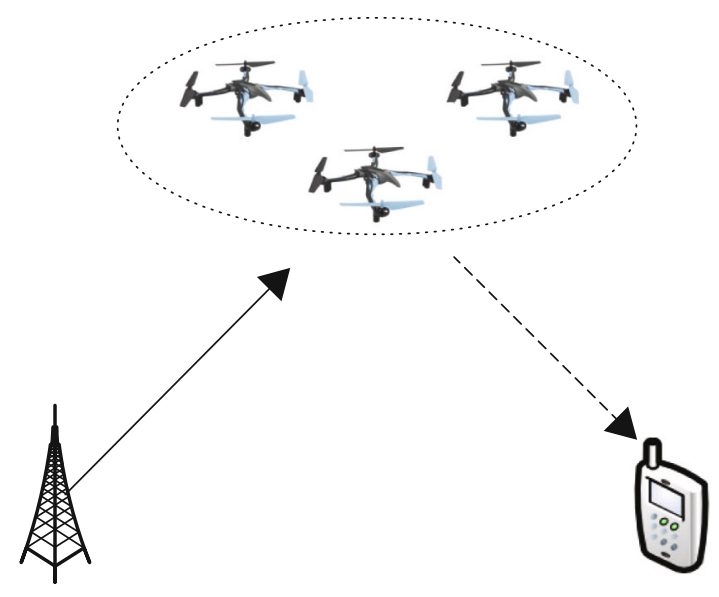

FIgURE 1: System model of cache-enabled amplify-and-forward UAV networks.

the selected UAV node will work as an amplify-andforward relaying node to help the data transmission. It is assumed that all links are block Rayleigh fading channel and independent with each other.

\section{Selection Algorithm and Performance Analysis}

3.1. Cached Links. If the requested file is cached by the UAV nodes, the power normalized transmission signal from the UAV node can be modeled as [13]

$$
s_{i}=\sqrt{\frac{1}{1+\kappa_{\eta}}}\left(\widehat{s}_{i}+\sqrt{\kappa_{\eta}} \eta_{i}\right),
$$

where $\widehat{s}_{i}$ is the information bearing signal with unit power, $\eta_{i} \in \mathscr{C} \mathcal{N}(0,1)$ is the distortion noise introduced by hardware impairments [22-25], and $\kappa_{\eta}$ is the hardware impairment distortion factor.

Since the classical MMSE channel estimation algorithm is adopted, there exists some estimation error between the real channel fading coefficient and the estimated version [26-29]. It is assumed that the estimation error is independent with the imperfect CSI [30-35]. Thus, the relationship between the imperfect channel status information and the ideal version is given as

$$
h_{u, i}=\sqrt{\frac{1}{1+\kappa_{e}}}\left(\widehat{h}_{u, i}+\sqrt{\kappa_{e} e_{i}}\right),
$$

where $h_{u, i} \in \mathscr{C} \mathcal{N}(0,1)$ is the CSI from the ith UAV node to the user, while $\widehat{h}_{u, i} \in \mathscr{C} \mathscr{N}(0,1)$ denotes the imperfect estimation of $h_{i}, e_{i} \in \mathscr{C} \mathscr{N}(0,1)$ is the difference between $\widehat{h}_{u, i}$ and $h_{u, i}$, and $\kappa_{e}$ is the channel estimation error factor. Specifically, $\kappa_{e}=0$ means the perfect channel estimation. 
Given that the $i$ th UAV relaying node is selected, the requested file is cached by the selected node. Then, the received signal of the user can be given as

$$
\begin{aligned}
y_{i} & =\sqrt{P_{R}} h_{u, i} s_{i}+n_{i} \\
& =\sqrt{\frac{P_{R}}{\left(1+\kappa_{e}\right)\left(1+\kappa_{\eta}\right)}}\left(\widehat{h}_{u, i}+\sqrt{\kappa_{e}} e_{i}\right)\left(\widehat{s}_{i}+\sqrt{\kappa_{\eta}} \eta_{i}\right)+n_{i},
\end{aligned}
$$

where $P_{R}$ is the transmission power of the UAV node, and $n_{i} \in \mathscr{C} \mathcal{N}\left(0, \sigma^{2}\right)$ is the received white Gauss noise.

Then, the signal to interference plus noise ratio (SINR) can be given as

$$
\gamma_{c, i}=\frac{\rho_{u, i}}{\rho_{u, i} \kappa_{\eta}+d_{1}}
$$

where

$$
\left\{\begin{array}{l}
\rho_{u, i}=\left|\widehat{h}_{u, i}\right|^{2}, \\
\lambda_{R}=\frac{P_{R}}{\sigma^{2}}, \\
d_{1}=\kappa_{e}\left(1+\kappa_{\eta}\right)+\left(1+\kappa_{e}\right) \frac{\left(1+\kappa_{\eta}\right)}{\lambda_{R}} .
\end{array}\right.
$$

Obviously, $\gamma_{c, i}$ is a monotone increasing function of $\rho_{u, i}$. In this case, the optimal UAV node can be given as follows:

$$
n_{1}^{*}=\arg \max _{i \in \Omega_{C}}\left(\rho_{u, i}\right) .
$$

Due to the independence between different $\rho_{u, i}$ 's, according to the ordered statistics, we have

$$
F_{\rho_{u, n_{1}^{*}}}(x)=\left[F_{\rho_{u, i}}(x)\right]^{N_{R}}=\left[1-e^{-x}\right]^{N_{R}},
$$

where $N_{R}$ is the number of relaying nodes.

Then, the outage probability of the cached links can be given as

$$
P_{O, C}=\operatorname{Pr}\left[C_{C}<C_{0}\right]=\operatorname{Pr}\left[\gamma_{r, n_{1}^{*}}<\gamma_{0}\right]=\operatorname{Pr}\left[\rho_{u, n_{1}^{*}}<\frac{d_{1} \gamma_{0}}{1-\kappa_{\eta} \gamma_{0}}\right],
$$

where $\gamma_{0}=2^{C_{0}}-1$ and $C_{0}$ are the required capacity.

Substituting (7) into (8), we obtain

$$
\begin{aligned}
P_{O, C} & =\operatorname{Pr}\left[\rho_{u, n_{1}^{*}}<\frac{d_{1} \gamma_{0}}{1-\kappa_{\eta} \gamma_{0}}\right] \\
& =\left[1-\exp \left(\frac{d_{1} \gamma_{0}}{1-\kappa_{\eta} \gamma_{0}}\right)\right]^{N_{R}}, \quad \gamma_{0}<\frac{1}{\kappa_{\eta}} .
\end{aligned}
$$

3.2. Relaying Links. On the other hand, if the requested file is not cached in the selected UAV node, the original message will be transmitted from the macro BS to the user with the help of the UAV node by using amplify-and-forward relaying protocol.

In this case, the received signal at the $i$ th UAV node can be given as

$$
r_{i}=h_{r, i} \sqrt{\frac{P_{S}}{1+\kappa_{\eta}}}\left(\widehat{s}_{i}+\sqrt{\kappa_{\eta}} \eta_{i}\right)+n_{r, i},
$$

where $P_{S}$ is the transmission power of the macro BS, $h_{r, i} \in$ $\mathscr{C} \mathcal{N}(0,1)$ denotes the channel fading coefficient from the macro BS to the $i$ th UAV node, and $n_{r, i} \in \mathscr{C} \mathcal{N}\left(0, \sigma^{2}\right)$ is the received Gauss noise at the UAV node.

Then, the UAV node will amplify the received signal with power $P_{R}$ and retransmit to the user. Thus, the received signal at the user through AF protocol can be given as

$$
y_{i}=h_{u, i} \zeta_{i} r_{i}+n_{i}
$$

where $\zeta_{i}$ is the power amplify factor as follows

$$
\zeta_{i}=\sqrt{\frac{P_{R}}{\rho_{r, i} P_{S}+\sigma^{2}}},
$$

and $\rho_{r, i}=\left|h_{r, i}\right|^{2}$ is the channel fading power of the first hop.

By substituting (2) and (12) into (11), we can obtain the SINR at the user through AF protocol

$$
\gamma_{r, i}=\frac{\rho_{r, i} \rho_{u, i}}{\rho_{r, i} \rho_{u, i} \kappa_{\eta}+\rho_{r, i} C_{r}+\rho_{u, i} C_{u}+C_{n}},
$$

where

$$
\left\{\begin{array}{l}
\rho_{r, i}=\left|\widehat{h}_{r, i}\right|^{2}, \\
\lambda_{S}=\frac{P_{S}}{\sigma^{2}}, \\
\lambda_{R}=\frac{P_{R}}{\sigma^{2}}, \\
c_{r}=\kappa_{e}\left(1+\kappa_{\eta}\right), \\
c_{u}=\frac{\left(1+\kappa_{e}\right)\left(1+\kappa_{\eta}\right)}{\lambda_{R}+\sigma^{2}}, \\
c_{n}=\kappa_{e} \sigma^{2}+\frac{\left(1+\kappa_{e}\right)\left(1+\kappa_{\eta}\right)}{\lambda_{S} / \lambda_{R}} .
\end{array} .\right.
$$

In order to obtain the best SINR, an UAV selection algorithm can be employed. Note that if the transmission power 
is large enough, i.e., $\lambda_{S} \rightarrow 0, \lambda_{R} \rightarrow 0$, the high order items can be ignored. Then, $\gamma_{r, i}$ in (13) can be simplified as

$$
\gamma_{r, i} \simeq\left[\kappa_{\eta}+\frac{c_{r}}{\rho_{u, i}}+\frac{c_{u}}{\rho_{r, i}}\right]^{-1} .
$$

To simply the complexity of theoretical analysis, we use the following approximation. Recall the following fact that [36]

$$
\frac{1}{\min (x, y)} \leq \frac{1}{x}+\frac{1}{y} \leq \frac{2}{\min (x, y)}, \quad \forall x, y>0
$$

To obtain the best SINR through AF protocol, the selection criterion can be given as

$$
n_{2}^{*}=\arg \max _{i \in \Omega_{R}} \min \left(\rho_{u, i}, \xi \rho_{r, i}\right)
$$

where $\xi=c_{r} / c_{u}$.

Theorem 1. The cumulative distribution functions of $\rho_{u, n_{2}^{*}}$ and $\rho_{r, n_{2}^{*}}$ are given as

$$
\left\{\begin{array}{l}
F_{\rho_{u, n_{2}^{*}}}(x)=N_{R} \sum_{k=1}^{N_{R}-1} b_{k}\left[\frac{1}{\xi_{2}}-\frac{e^{-x}}{\xi_{3}}+\frac{e^{-\xi_{2} x}}{\left(\xi_{3} \xi_{2}\right)}\right], \\
F_{\rho_{r, n_{2}^{*}}}(x)=N_{R} \sum_{k=1}^{N_{R}-1} b_{k}\left[\frac{1}{\xi_{2}}-\frac{e^{-x}}{\xi_{4}}+\frac{e^{-\xi_{2} \xi x}}{\left(\xi_{2} \xi_{4}\right)}\right],
\end{array}\right.
$$

for $N_{R} \geq 2$.

$$
F_{\rho_{u, n_{2}^{*}}}(x)=F_{\rho_{r, n_{2}^{*}}}(x)=1-e^{-x}
$$

for $N_{R}=1$, where

$$
\left\{\begin{array}{l}
\xi_{1}=1+\frac{1}{\xi}, \\
\xi_{2}=(k+1) \xi_{1}, \\
\xi_{3}=k \xi_{1}+\frac{1}{\xi}=\xi_{2}-1, \\
\xi_{4}=k \xi_{1}+1=\xi_{2}-\frac{1}{\xi}, \\
b_{k}=C_{N_{R-1}}^{k}(-1)^{k-1} k \xi_{1} .
\end{array}\right.
$$

Taking the derivative of equations in Theorem 1, we can obtain the following corollary.
Corollary 2. The probability distribution functions of $\rho_{u, n_{2}^{*}}$ and $\rho_{r, n_{2}^{*}}$ are given as

$$
\left\{\begin{array}{l}
f_{\rho_{u, n_{2}^{*}}}(x)=N_{R} \sum_{k=1}^{N_{R}-1} \frac{b_{k}}{\xi_{3}\left(e^{-x}-e^{-\xi_{2} x}\right)}, \\
f_{\rho_{r, n_{2}^{*}}}(x)=N_{R} \sum_{k=1}^{N_{R}-1} \frac{b_{k}}{\xi_{4}\left(e^{-x}-e^{-\xi_{2} \xi x}\right)},
\end{array}\right.
$$

for $N_{R} \geq 2$, and

$$
f_{\rho_{u, n_{2}^{*}}}(x)=f_{\rho_{r, n_{2}^{*}}}(x)=e^{-x}
$$

for $N_{R}=1$.

Proof. See Appendix VI-A.

Considering the SINR expression in (13), the outage occurs when $\gamma_{r, n_{2}^{*}}$ falls below the quality-of-service requirement, i.e.,

$$
P_{O, R}=\operatorname{Pr}\left[C_{R}<C_{0}\right]=\operatorname{Pr}\left[\gamma_{r, n_{2}^{*}}<\gamma_{0}\right]
$$

where $\gamma_{0}=2^{C_{0}}-1$.

Substituting (13) into (24), we have

$$
\begin{aligned}
P_{O, R} & =\operatorname{Pr}\left[\frac{\rho_{r, i} \rho_{u, i}}{\rho_{r, i} \rho_{u, i} \kappa_{\eta}+\rho_{r, i} C_{r}+\rho_{u, i} C_{u}+C_{n}}<\gamma_{0}\right] \\
& =\operatorname{Pr}\left[\rho_{r, i} \rho_{u, i}\left(1-\gamma_{0} \kappa_{\eta}\right)<\left(\rho_{r, i} C_{r} \gamma_{0}+\rho_{u, i} C_{u} \gamma_{0}+\gamma_{0} C_{n}\right)\right] \\
& =\operatorname{Pr}\left[\rho_{r, i}\left[\rho_{u, i}\left(1-\gamma_{0} \kappa_{\eta}\right)-C_{r} \gamma_{0}\right]<\left(\rho_{u, i} C_{u} \gamma_{0}+\gamma_{0} C_{n}\right)\right] .
\end{aligned}
$$

Applying conditional probability formula, we have

$$
\begin{aligned}
P_{O, R}= & \operatorname{Pr}\left[\rho_{r, i}\left[\rho_{u, i}\left(1-\gamma_{0} \kappa_{\eta}\right)-c_{r} \gamma_{0}\right]<\left(\rho_{u, i} c_{u} \gamma_{0}+\gamma_{0} c_{n}\right)\right] \\
= & \operatorname{Pr}\left[\rho_{u, i}<c_{1}\right]+\operatorname{Pr}\left[\rho_{r, i}<c_{2} \frac{\rho_{u, i}+c_{n} / c_{u}}{\rho_{u, i}-c_{1}}, \rho_{u, i} \geq c_{1}\right] \\
= & \underbrace{\operatorname{Pr}\left[\rho_{u, i}<c_{1}\right]}_{P_{1}}+\underbrace{\operatorname{Pr}\left[\rho_{r, i} \leq c_{2}, \rho_{u, i} \geq c_{1}\right]}_{P_{2}} \\
& +\underbrace{\operatorname{Pr}\left[c_{2}<\rho_{r, i}<c_{2} \frac{\rho_{u, i}+c_{n} / c_{u}}{\rho_{u, i}-c_{1}}, \rho_{u, i} \geq c_{1}\right]}_{P_{3}},
\end{aligned}
$$


where

$$
\left\{\begin{array}{l}
c_{1}=\frac{c_{r} \gamma_{0}}{\left(1-\gamma_{0} \kappa_{\eta}\right)}, \\
c_{2}=\frac{c_{u} \gamma_{0}}{\left(1-\gamma_{0} \kappa_{\eta}\right)}, \\
c_{3}=\frac{c_{n}}{\left(c_{1} c_{u}\right)} .
\end{array}\right.
$$

Applying (18) on (25), we can obtain

$$
\begin{aligned}
& P_{1}=F_{\rho_{u, n_{2}^{*}}}\left(c_{1}\right), \\
& P_{2}=F_{\rho_{r, n_{2}^{*}}}\left(c_{2}\right)\left[1-F_{\rho_{u, n_{2}^{*}}}\left(c_{1}\right)\right] .
\end{aligned}
$$

To derive the exact expression of $P_{3}$, firstly we give the following definition

$$
\begin{aligned}
F_{Z}(z) & =\operatorname{Pr}\left[\frac{\rho_{u, i}+c_{n} / c_{u}}{\rho_{u, i}-c_{1}}<z, \rho_{u, i} \geq c_{1}\right], \quad z>1 \\
& =\operatorname{Pr}\left[\rho_{u, i}>c_{1} \frac{z+c_{n} /\left(c_{1} c_{u}\right)}{z-1}, \rho_{u, i} \geq c_{1}\right], \quad z>1 \\
& =\operatorname{Pr}\left[\rho_{u, i}>c_{1} \frac{z+c_{n} /\left(c_{1} c_{u}\right)}{z-1}\right], \quad z>1 \\
& =\operatorname{Pr}\left[\rho_{u, i}>c_{1} \frac{z+c_{3}}{z-1}\right], \quad z>1 .
\end{aligned}
$$

Applying (18) on (29), we can obtain

$$
F_{Z}(z)=\operatorname{Pr}\left[\rho_{u, i}>c_{1} \frac{z+c_{3}}{z-1}\right], \quad z>1=F_{\rho_{u, n_{2}^{*}}}\left(c_{1} \frac{z+c_{3}}{z-1}\right) .
$$

Thus, the item $P_{3}$ in equation (25) can be given as

$$
\begin{aligned}
P_{3} & =\operatorname{Pr}\left[c_{2}<\rho_{r, i}<c_{2} \frac{\rho_{u, i}+c_{n} / c_{u}}{\rho_{u, i}-c_{1}}, \rho_{u, i} \geq c_{1}\right] \\
& =\int_{x>c_{2}}^{+\infty} f_{\rho_{r, n_{2}^{*}}}(x)\left[1-F_{Z}\left(\frac{x}{c_{2}}\right)\right] d x \\
& =\int_{x>c_{2}}^{+\infty} f_{\rho_{r, n_{2}^{*}}}(x)\left[1-F_{\rho_{u, n_{2}^{*}}}\left(c_{1} \frac{x+c_{3} c_{2}}{x-c_{2}}\right)\right] d x .
\end{aligned}
$$

By using necessary mathematical derivation and applying equation (3.324-1) in [37], i.e.,

$$
\int_{0}^{+\infty} \exp \left(\frac{\beta}{4 x}-\gamma x\right) d x=\sqrt{\frac{\beta}{\gamma}} K_{1}(\sqrt{\beta \gamma}),
$$

the closed-form expression of $P_{3}$ can be obtained.

By substituting (27), (28), and (31) into (25), we can obtain the exact closed-form expression of $P_{O, R}$.

\section{Asymptotical Analysis}

To get a deep insight on the impact of hardware impairments with imperfect channel estimation, we will conduct the asymptotical analysis of outage probability. Note that if the transmission power is large enough, i.e., $1 / \lambda_{S} \rightarrow 0,1 / \lambda_{R} \rightarrow 0$, and $\kappa_{e} \rightarrow 0, \kappa_{\eta} \rightarrow 0$.

Thus, we have

$$
\begin{aligned}
& \left\{\begin{array}{l}
c_{r} \simeq \kappa_{e}\left(1+\kappa_{\eta}\right), \\
c_{u} \simeq \sigma^{2}, \\
c_{n} \simeq \kappa_{e} \sigma^{2} \\
d_{1} \simeq \kappa_{e}\left(1+\kappa_{\eta}\right), \\
\xi \simeq \frac{\kappa_{e}\left(1+\kappa_{\eta}\right)}{\sigma^{2}},
\end{array}\right. \\
& \left\{\begin{array}{l}
c_{1} \simeq \frac{\kappa_{e}\left(1+\kappa_{\eta}\right) \gamma_{0}}{\left(1-\gamma_{0} \kappa_{\eta}\right)}, \\
c_{2} \simeq \frac{\sigma^{2} \gamma_{0}}{\left(1-\gamma_{0} \kappa_{\eta}\right)}, \\
c_{3} \simeq \frac{\left(1-\gamma_{0} \kappa_{\eta}\right)}{\left(1+\kappa_{\eta}\right) \gamma_{0}} .
\end{array}\right.
\end{aligned}
$$

Applying the approximation that $1-e^{-x} \simeq x$, if $x \rightarrow 0$ on (9), we have

$$
P_{O, C} \simeq\left(\frac{d_{1} \gamma_{0}}{1-\kappa_{\eta} \gamma_{0}}\right)^{N_{C}} \simeq\left[\gamma_{0} \frac{\kappa_{e}\left(1+\kappa_{\eta}\right)}{1-\kappa_{\eta} \gamma_{0}}\right]^{N_{C}} .
$$

According to the asymptotical expression on outage probability for cached links, we can conclude the following remarks:

Remark 3. There is an error floor for the cached links when the transmission power is large enough. Meanwhile, the diversity order with respect to the transmission power is zero.

Remark 4. The error floor for the cached links is dependent on the channel estimation error factor $\kappa_{e}$, the hardware impairment distortion factor $\kappa_{\eta}$, and the QoS requirements $\gamma_{0}$.

Remark 5. The diversity order of error floor for the cached links is equal to the number of cached UAV nodes.

Considering the SINR expression in (15), we have

$$
\begin{aligned}
P_{O, R} & \simeq \operatorname{Pr}\left[\max _{i \in \Omega_{R}} \min \left(\rho_{u, i}, \xi \rho_{r, i}\right)<\gamma_{0} \frac{\xi c_{u}}{\left(1-\gamma_{0} \kappa_{\eta}\right)}\right] \\
& =\left\{\operatorname{Pr}\left[\min \left(\rho_{u, i}, \xi \rho_{r, i}\right)<c_{4}\right]\right\}^{N_{R}} .
\end{aligned}
$$




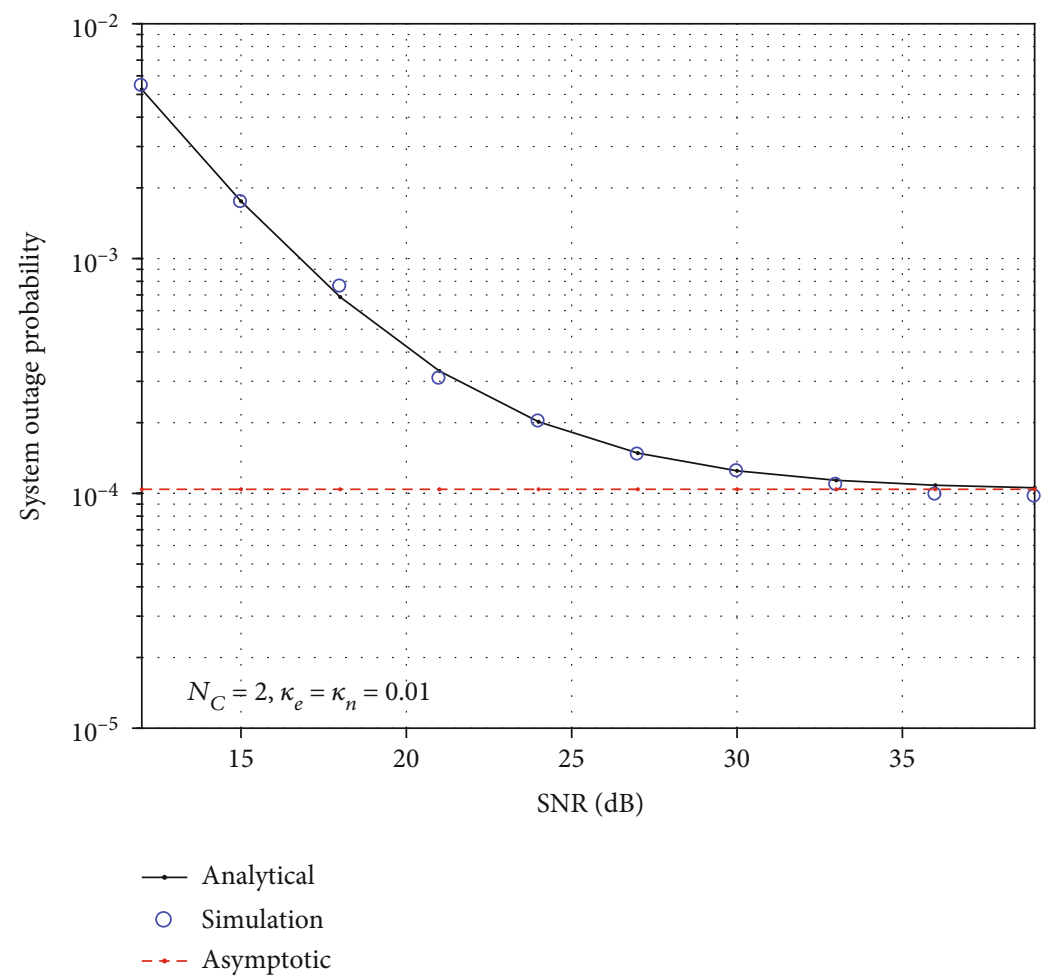

FIgURE 2: Outage probability for cached link with $N_{C}=2$.

By using the result in (A.3), we have

$$
\begin{aligned}
P_{O, R} & \simeq\left(1-e^{-\xi_{1} c_{4}}\right)^{N_{R}} \simeq\left(\xi_{1} c_{4}\right)^{N_{R}} \simeq\left[\gamma_{0} \frac{(1+\xi) \sigma^{2}}{\left(1-\gamma_{0} \kappa_{\eta}\right)}\right]^{N_{R}} \\
& \simeq\left[\gamma_{0} \frac{\sigma^{2}+\kappa_{e}\left(1+\kappa_{\eta}\right)}{\left(1-\gamma_{0} \kappa_{\eta}\right)}\right]^{N_{R}},
\end{aligned}
$$

where

$$
c_{4}=\gamma_{0} \frac{\xi c_{u}}{\left(1-\gamma_{0} \kappa_{\eta}\right)} \simeq \gamma_{0} \frac{\xi \sigma^{2}}{\left(1-\gamma_{0} \kappa_{\eta}\right)} .
$$

Remark 6. There also exists an error floor for the relaying links when the transmission power is large enough.

Remark 7. The diversity order of error floor for the relaying links is equal to the number of relaying UAV nodes.

\section{Simulation Results}

In this section, simulation results are provided to verify the accuracy of the theoretical analysis. For the sake of simplicity, we set $\kappa_{e}=\kappa_{\eta}=\sigma^{2}$. The impacts of the system parameters, such as the number of UAV nodes $N$, the transmission SNR of the cached links and the relaying links $\lambda_{S}, \lambda_{R}$, and the capacity threshold $C_{0}$, are investigated.

Figure 2 shows the impacts of transmission $\operatorname{SNR} \lambda_{S}$ on outage performance for cached links. In this simulation, we have the following system parameters: $N_{C}=2, \kappa_{e}=\kappa_{\eta}=0.01$, $C_{0}=1 \mathrm{bps} / \mathrm{Hz}$, and the transmission SNR $\lambda_{S}$ changes from $12 \mathrm{~dB}$ to $39 \mathrm{~dB}$. The simulation results and the theoretical analysis as well as the asymptotic analysis are compared in this figure. As shown in this figure, in all SNR regions, the simulation results match well with the theoretical analysis. Furthermore, an error floor appears when the transmission SNR grows large, which coincides with the asymptotic performance analysis. The reason is that when SNR is large enough, the bottleneck of the system performance is the hardware impairments and the channel estimation error. The detailed relationship between the error floor and $\kappa_{\eta}$ and $\kappa_{e}$ is given as in equations (34) and (36). When the requested file is not hit by the UAV nodes, amplify-andforward protocol is adopted and the simulation results are present in Figure 3, where similar conclusion can be obtained for relaying links with $N_{R}=2$.

Figures 4 and 5 show the effects of the capacity threshold $C_{0}$ on outage probability for cached links and relaying links, respectively. The system parameters are set as follows: $N_{C}=N_{R}=2, \kappa_{e}=\kappa_{\eta}=0.01$, and the capacity threshold $C_{0}$ changed from $1 \mathrm{bps} / \mathrm{Hz}$ to $3 \mathrm{bps} / \mathrm{Hz}$. From the two figures, we can observe that $C_{0}$ shows significant impact on outage probability for all of the SNR regions. Specifically, the diversity order of outage probability is zero with respect to the transmission SNR $\lambda_{S}$ or $\lambda_{R}$.

Figures 6 and 7 show the effects of the hardware impairment factor $\kappa_{\eta}$ on outage probability for cached links and relaying links, respectively. The system parameters are set as follows: $N_{C}=N_{R}=2, \kappa_{e}=\kappa_{\eta}=0.01, C_{0}=1 \mathrm{bps} / \mathrm{Hz}$, and 


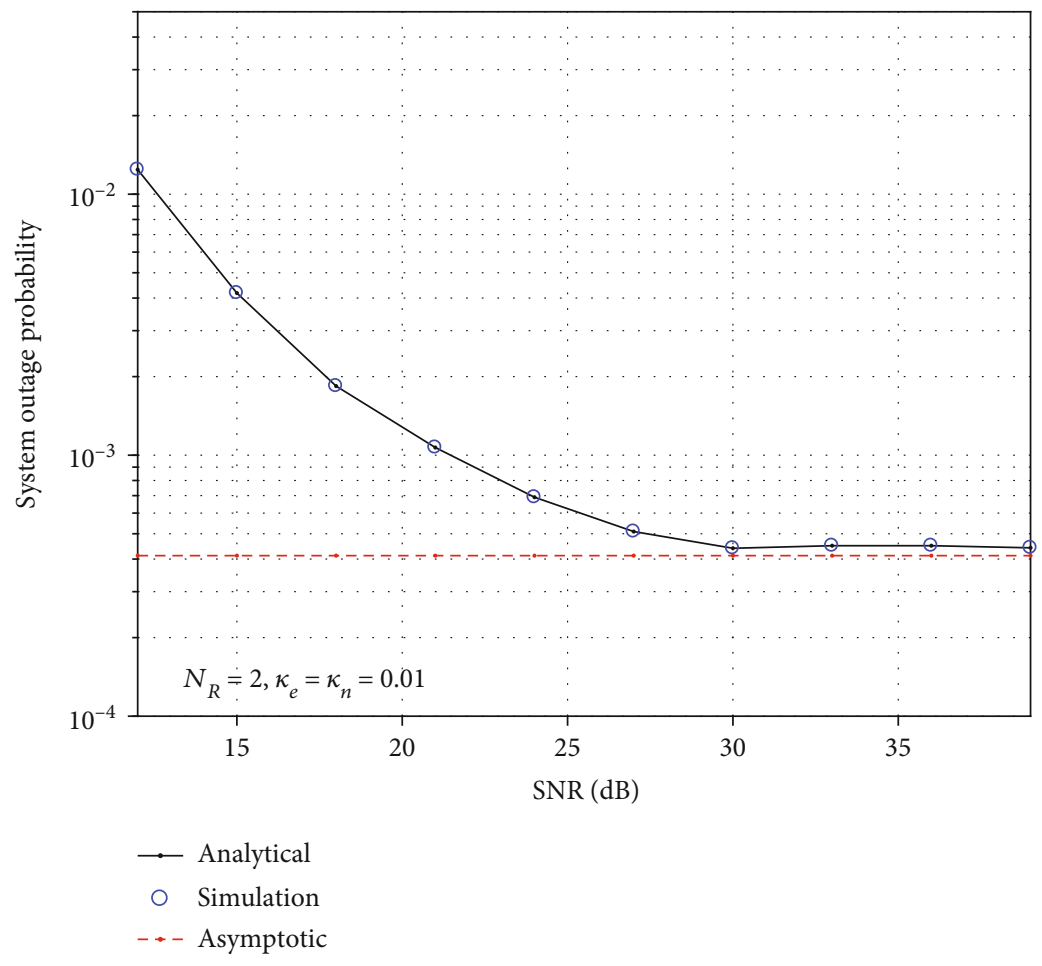

FIgURE 3: Outage probability for AF relaying links with $N_{R}=2$.

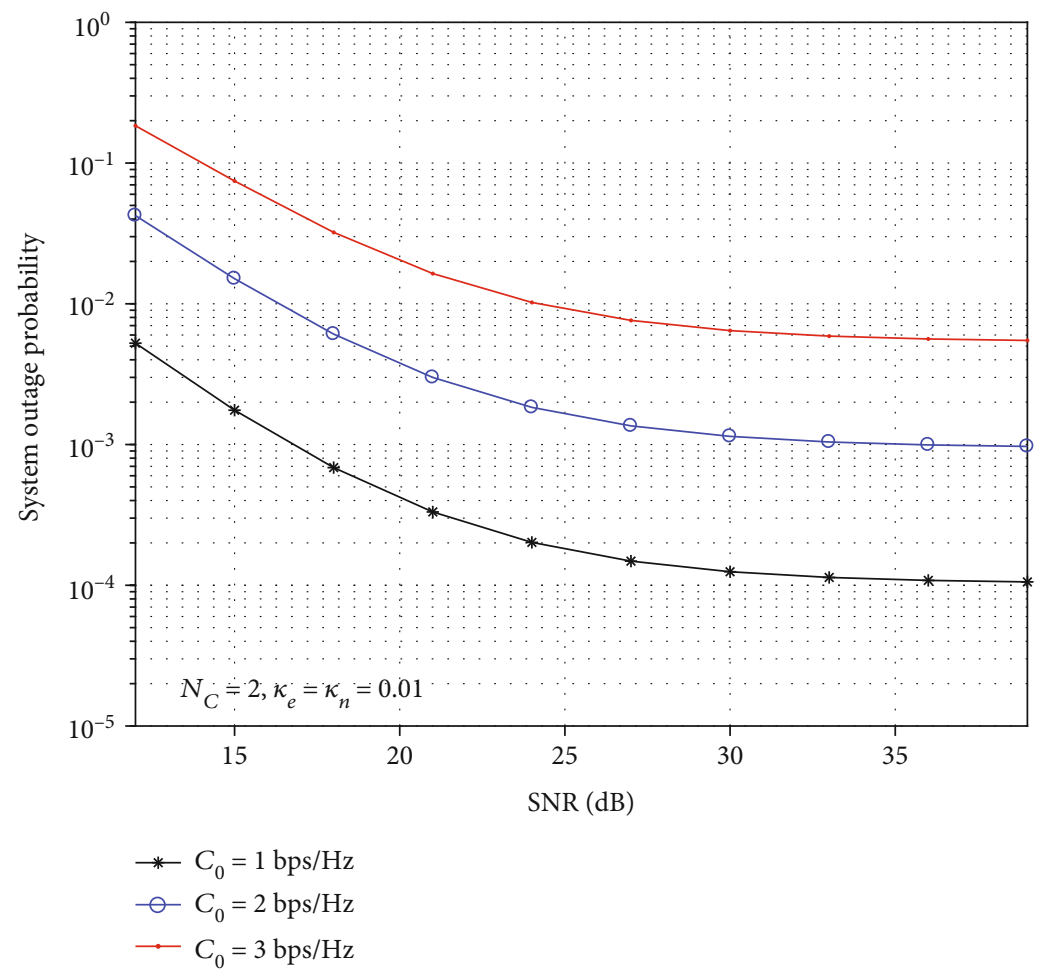

FIgURE 4: Outage probability versus capacity threshold for cached links with $N_{C}=2$.

the hardware impairment factor $\kappa_{\eta}$ changed from 0.01 to 0.03 . From these figures, we can observe that $C_{0}$ shows considerable impact on outage probability especially in high
SNR regions. Specifically, the larger $\kappa_{\eta}$ will introduce higher error floor. The reason is that, in the considered scenario, the bottleneck of the system performance is the hardware 


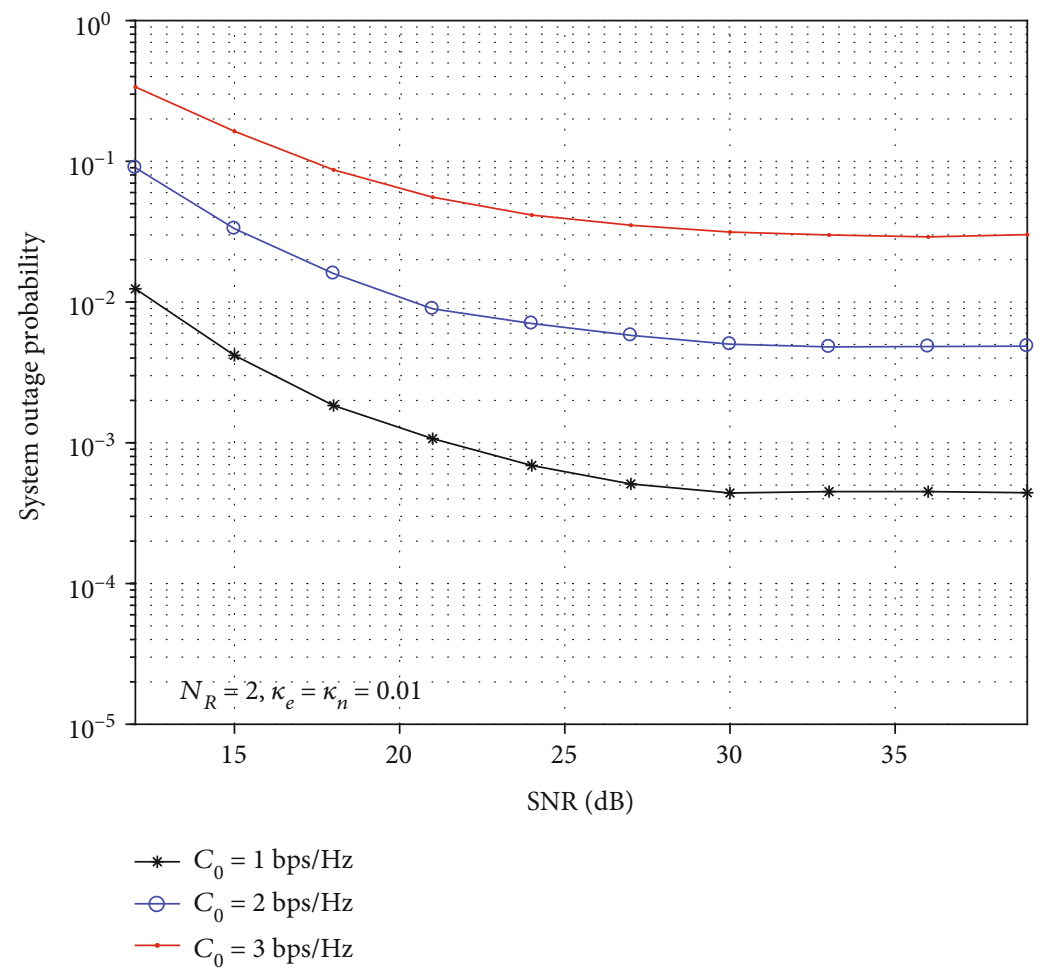

FIgURE 5: Outage probability versus capacity threshold for relaying links with $N_{R}=2$.

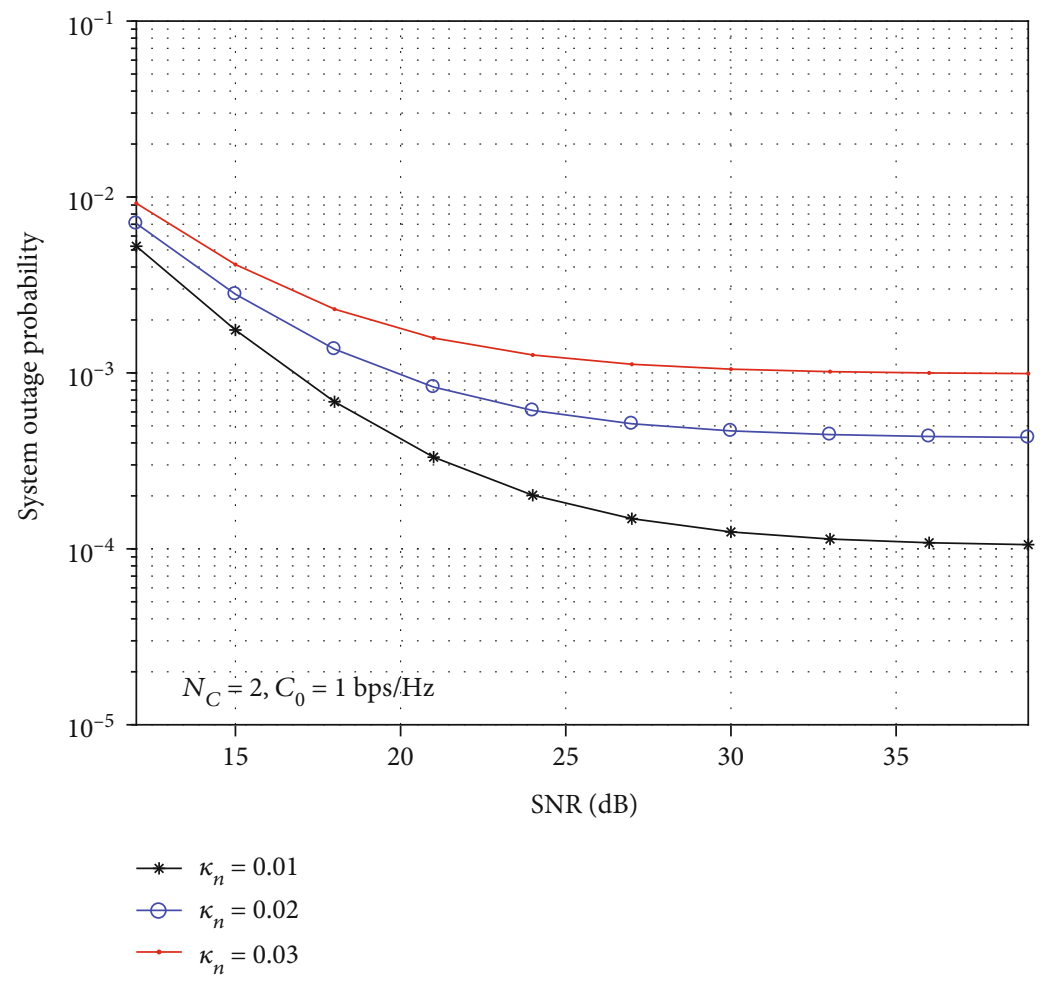

FIgURE 6: Outage probability versus $\kappa_{\eta}$ for cached links with $N_{C}=2$.

impairments. When the hardware impairment factor $\kappa_{\eta}$ grows larger, the noise power will be higher, which will deteriorate the system performance.
The effect of the number of cached UAV nodes is present in Figure 8, where the system parameters are set as follows: $\kappa_{e}=\kappa_{\eta}=0.01, C_{0}=1 \mathrm{bps} / \mathrm{Hz}$, and the number of cached 


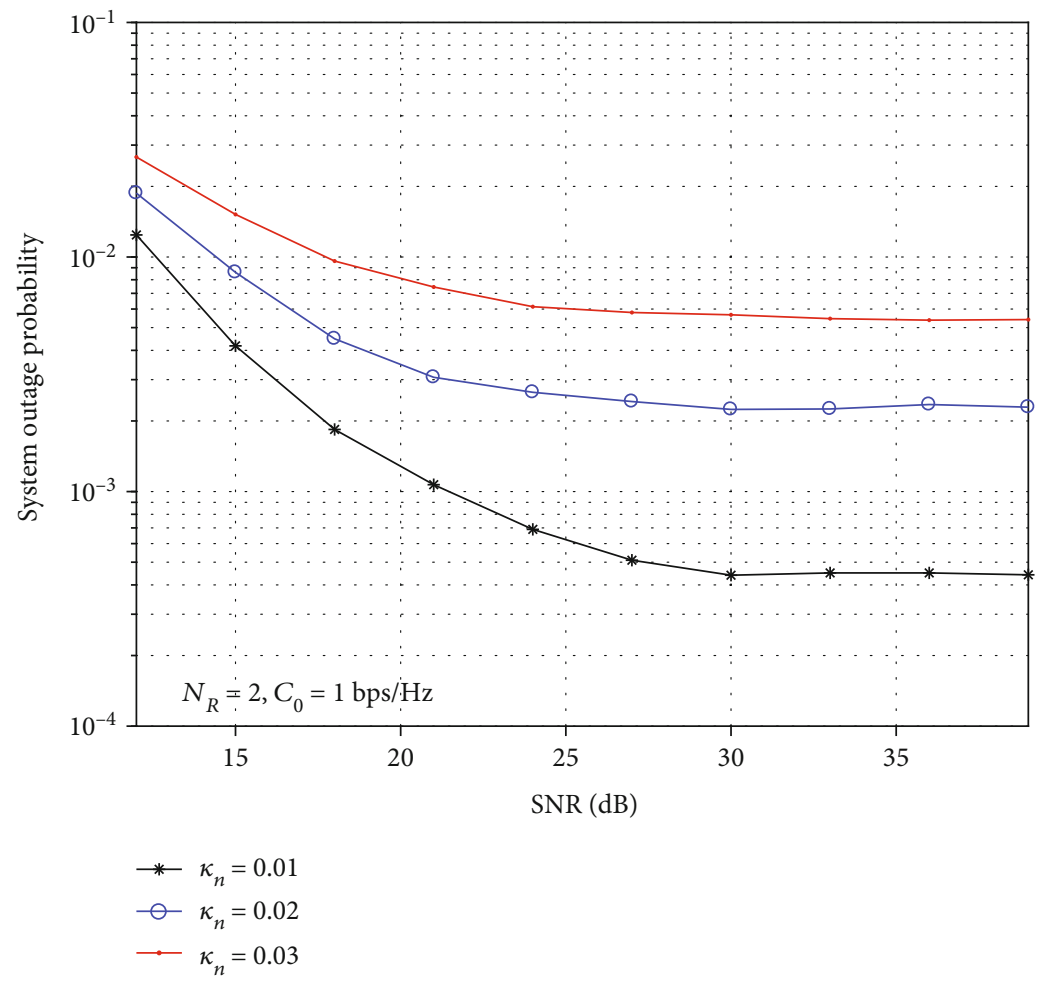

Figure 7: Outage probability versus $\kappa_{\eta}$ for relaying links with $N_{R}=2$.

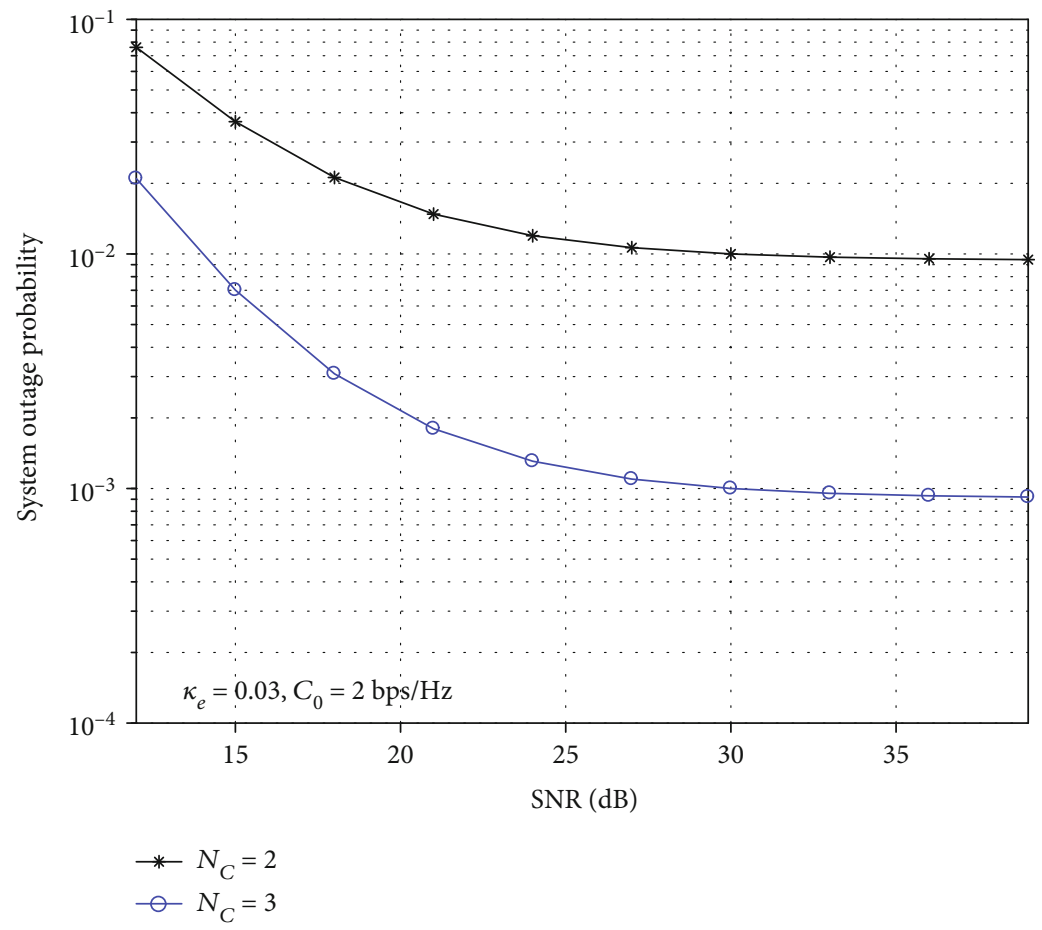

FIgURE 8: Outage probability versus $N_{C}$ for cached links.

UAV nodes $N_{C}$ changed from 2 to 3 . We can see from this figure that $N_{C}$ shows great impact on system performance. As mentioned by the remarks of asymptotic anal- ysis, the diversity order of error floor for the cached/ relaying links is equal to the number of cached/relaying UAV nodes. 


\section{Conclusions}

The impacts of hardware impairments with imperfect channel estimation for cache-enabled UAV relaying networks are investigated in this paper. To improve the QoS of the wireless channels, the best cached UAV nodes or AF relaying nodes are selected to help the message transmission from the macro BS to the user. The impacts of the setup parameters, such as the number of cached/relaying nodes, the hardware impairment factor, the channel estimation error, and the transmission SNR, on the outage probability are present by deriving the exact closed-form expressions on outage probability. Also, the asymptotic results are present when the transmission SNR is large enough. From the asymptotic analysis, we can see that there is an error floor introduced by the hardware impairments and imperfect channel estimation. Specifically, the error floor is dependent on the channel estimation error factor, the hardware impairment distortion factor, and the QoS requirements. In future works, we will introduce deep learning-based [38-40] or Q-learning-based algorithms [41, 42] to further improve the system performance. Moreover, we will apply the considered wireless techniques into some practical IoT systems $[43,44]$ to achieve the green energy applications.

\section{Appendix}

\section{Proof of Theorem 1}

For the case $N_{R}=1$, the theorem obviously holds.

We will focus on the case that $N_{R} \geq 2$. Firstly, we give the following definition

$$
z_{i}=\min \left(\rho_{u, i}, \xi \rho_{r, i}\right)
$$

Then, the PDF of $z_{i}$ can be derived as

$$
\begin{aligned}
F_{z i}(x) & =\operatorname{Pr}\left\{\min \left(\rho_{u, i}, \xi \rho_{r, i}\right)<z\right\} \\
& =1-\operatorname{Pr}\left\{\min \left(\rho_{u, i}, \xi \rho_{r, i}\right) \geq z\right\} \\
& =1-\operatorname{Pr}\left\{\rho_{u, i} \geq z, \rho_{r, i} \geq \frac{z}{\xi}\right\}
\end{aligned}
$$

Due to the independence between $\rho_{u, i}$ and $\rho_{r, i}$, we obtain

$F_{z i}(x)=1-\operatorname{Pr}\left\{\rho_{u, i} \geq z\right\} \operatorname{Pr}\left\{\rho_{r, i} \geq \frac{z}{\xi}\right\}=1-\frac{e^{-z} e^{-z}}{\xi=1-e^{-(1+1 / \xi) z}}$.

Taking the derivative of $F_{z_{i}}(x)$, we can get the PDF of $z_{i}$ as

$$
f_{z i}(x)=\xi_{1} e^{-\xi_{1} x}
$$

where

$$
\xi_{1}=1+\frac{1}{\xi}
$$

Secondly, we give another definition as

$$
\theta_{m}=\max _{i \in \Omega_{R} / m} z_{i}
$$

Since $z_{i}$ 's are independent identical distribution, we can have

$$
F_{\theta_{m}}(x)=\left[F_{z_{i}}(x)\right]^{N_{R}-1}
$$

By substituting (A.3) into (A.7) and applying the binomial theorem, we have

$$
\begin{aligned}
F_{\theta_{m}}(x) & =\left(1-e^{-\xi_{1} z}\right)^{N_{R}-1} \\
& =\sum_{k=0}^{N_{R}-1} C_{N_{R}-1}^{k}(-1)^{k} e^{-k \xi_{1} z} \\
& =1-\sum_{k=1}^{N_{R}-1} C_{N_{R}-1}^{k}(-1)^{k-1} e^{-k \xi_{1} z}, \quad N_{R} \geq 2 .
\end{aligned}
$$

As such, the PDF of $\theta_{m}$ can be obtained as

$$
f_{\theta_{m}}(x)=\sum_{k=1}^{N_{R}-1} b_{k} e^{-k \xi_{1} x}
$$

where $b_{k}=C_{N_{R}-1}^{k}(-1)^{k-1} k \xi_{1}$.

According to the definition in (17), we can derive the CDF of $\rho_{u, n_{2}^{*}}$,

$$
\begin{aligned}
F_{\rho_{u, n_{2}^{*}}}(x) & =\sum_{m=1}^{N_{R}} \operatorname{Pr}\left[\rho_{u, m}<x, z_{m}>\theta_{m}\right] \\
& =N_{R} \operatorname{Pr}\left[\rho_{u, 1}<x, z_{1}>\theta_{1}\right] \\
& =N_{R} \operatorname{Pr}\left[\rho_{u, 1}<x, \rho_{u, 1}>\theta_{1}, \xi \rho_{r, 1}>\theta_{1}\right] \\
& =N_{R} \operatorname{Pr}\left[\theta_{1}<\rho_{u, 1}<x, \rho_{r, 1}>\frac{\theta_{1}}{\xi}, \theta_{1}<x\right] .
\end{aligned}
$$

By using the result in (A.9), we have

$$
\begin{aligned}
F_{\rho_{u, n_{2}^{*}}}(x) & =N_{R} \operatorname{Pr}\left[\theta_{1}<\rho_{u, 1}<x, \rho_{r, 1}>\frac{\theta_{1}}{\xi}, \theta_{1}<x\right] \\
& =N_{R} \int_{0}^{x} f_{\theta_{1}}(\theta)\left(e^{-\theta}-e^{-x}\right) e^{-\theta / \xi} d \theta \\
& =N_{R} \sum_{k=1}^{N_{R}-1} b_{k}\left[\frac{1}{\xi_{2}}-\frac{e^{-x}}{\xi_{3}}+\frac{e^{-\xi_{2} x}}{\xi_{3} \xi_{2}}\right]
\end{aligned}
$$


where

$$
\left\{\begin{array}{l}
\xi_{2}=(k+1) \xi_{1}, \\
\xi_{3}=k \xi_{1}+\frac{1}{\xi}=\xi_{2}-1, \\
\xi_{4}=k \xi_{1}+1=\xi_{2}-\frac{1}{\xi} .
\end{array}\right.
$$

Similarly, we can obtain the $\mathrm{CDF}$ of $\rho_{r, n_{2}^{*}}$ as follows:

$$
F_{\rho_{r, n_{2}^{*}}}(x)=N_{R} \sum_{k=1}^{N_{R}-1} b_{k}\left[\frac{1}{\xi_{2}}-\frac{e^{-x}}{\xi_{4}}+\frac{e^{-\xi_{2} \xi x}}{\xi \xi_{2} \xi_{4}}\right] .
$$

Thus, Theorem 1 is proved.

\section{Data Availability}

The data of this work can be available through the request on the corresponding author by e-mail.

\section{Conflicts of Interest}

The authors declare that there is no conflict of interest regarding the publication of this paper.

\section{Acknowledgments}

This work was partly supported by the Natural Science Foundation of Guangdong Province with grant number 2018A030313736, the Scientific Research Project of Education Department of Guangdong with grant numbers 2017GKTSCX045 and 2019GZDXM002, the Project of Technology Development Foundation of Guangdong with grant number 706049150203, the Science and Technology Program of Guangzhou under grant 201807010103, the Application Technology Collaborative Innovation Center of GZPYP with grant number 2020ZX01, the Yangcheng Scholar, Scientific Research Project of Guangzhou Education Bureau with grant number 202032761, the Department of Education of Guangdong Province of China with grant number 2017GKTSCX047, the Education Department of Guangzhou City of China with grant number 201831785, the Personnel Training Project of Guangzhou University with grant number RP2020122, and the Yangcheng Scholars Innovative Academic Team Project with grant number 1201610010.

\section{References}

[1] J. Xia, C. Li, X. Lai et al., "Cache-aided mobile edge computing for $\mathrm{B}_{5} \mathrm{G}$ wireless communication networks," EURASIP Journal on Wireless Communications and Networking, vol. 2020, no. 1, 2020.

[2] C. Li, Z. Gao, J. Xia, D. Deng, and L. Fan, "Cache-enabled physical-layer secure game against smart uavassisted attacks in $b_{5}$ g NOMA networks," EURASIP Journal on Wireless Communications and Networking, vol. 2020, no. 1, 2020.
[3] J. Zhao, X. Guan, and X. P. Li, "Power allocation based on genetic simulated annealing algorithm in cognitive radio networks," Chinese Journal of Electronics, vol. 22, no. 1, pp. 177180, 2013.

[4] Y. Guo, Z. Zhao, R. Zhao et al., "Intelligent offloading strategy design for relaying mobile edge computing networks," IEEE Access, vol. 8, pp. 35127-35135, 2020.

[5] D. Deng, J. Xia, L. Fan, and X. Li, "Link selection in bufferaided cooperative networks for green iot," IEEE Access, vol. 8, pp. 30763-30771, 2020.

[6] J. Zhao, S. Ni, L. Yang, Z. Zhang, Y. Gong, and X. You, "Multiband cooperation for 5G HetNets: a promising network paradigm," IEEE Vehicular Technology Magazine, vol. 14, no. 4, pp. 85-93, 2019.

[7] J. Zhu, C. Gong, S. Zhang, M. Zhao, and W. Zhou, "Foundation study on wireless big data: concept, mining, learning and practices," China Ccommunications, vol. 15, no. 12, pp. 1-15, 2018.

[8] D. Deng and J. Xia, "Cache-enabled cooperative edge networks for intelligent connected vehicles," IEEE Access, vol. 7, pp. 166939-166949, 2019.

[9] Z. Ming, S. Zhou, W. Zhou, and J. Zhu, "An improved uplink sparse coded multiple access," IEEE Communications Letters, vol. 21, no. 1, pp. 176-179, 2017.

[10] Y. Wang, M. Zhao, D. Deng, S. Zhou, and W. Zhou, "Fractional sparse code multiple access and its optimization," IEEE Wireless Communications Letters, vol. 7, no. 6, pp. 990-993, 2018.

[11] J. Song, H. Song, and W. Choi, "Which one is better to cache: requested contents or interfering contents?," IEEE Wireless Communications Letters, vol. 8, no. 3, pp. 861-864, 2019.

[12] D. Ko, B. Hong, and W. Choi, "Probabilistic caching based on maximum distance separable code in a user-centric clustered cache-aided wireless network," IEEE Transactions on Wireless Communications, vol. 18, no. 3, pp. 1792-1804, 2019.

[13] X. Li, J. Li, Y. Liu, Z. Ding, and A. Nallanathan, "Residual transceiver hardware impairments on cooperative NOMA networks," IEEE Transactions on Wireless Communications, vol. 19, no. 1, pp. 680-695, 2020.

[14] O. T. Demir and E. Bjornson, "Channel estimation in massive MIMO under hardware non-linearities: Bayesian methods versus deep learning," IEEE Open Journal of the Communications Society, vol. 1, pp. 109-124, 2020.

[15] J. Zhu, D. W. K. Ng, N. Wang, R. Schober, and V. K. Bhargava, "Analysis and design of secure massive MIMO systems in the presence of hardware impairments," IEEE Transactions on Wireless Communications, vol. 16, no. 3, pp. 2001-2016, 2017.

[16] A. K. Papazafeiropoulos, S. K. Sharma, S. Chatzinotas, and B. Ottersten, "Ergodic capacity analysis of AF DH MIMO relay systems with residual transceiver hardware impairments: conventional and large system limits," IEEE Transactions on Vehicular Technology, vol. 66, no. 8, pp. 7010-7025, 2017.

[17] E. Björnson, J. Hoydis, M. Kountouris, and M. Debbah, "Massive MIMO systems with non-ideal hardware: energy efficiency, estimation, and capacity limits," IEEE Transactions on Information Theory, vol. 60, no. 11, pp. 7112 7139, 2014.

[18] G. Ding, X. Gao, Z. Xue, Y. Wu, and Q. Shi, "Massive mimo for distributed detection with transceiver impairments," IEEE Transactions on Vehicular Technology, vol. 67, no. 1, pp. 604-617, 2018. 
[19] S. Guo and X. Zhou, "Robust resource allocation with imperfect channel estimation in NOMA-based heterogeneous vehicular networks," IEEE Transactions on Communications, vol. 67, no. 3, pp. 2321-2332, 2019.

[20] W. A. Al-Hussaibi and F. H. Ali, "A closed-form approximation of correlated multiuser mimo ergodic capacity with antenna selection and imperfect channel estimation," IEEE Transactions on Vehicular Technology, vol. 67, no. 6, pp. 5515-5519, 2018.

[21] Y. M. Khattabi and M. M. Matalgah, "Alamouti-OSTBC wireless cooperative networks with mobile nodes and imperfect CSI estimation," IEEE Transactions on Vehicular Technology, vol. 67, no. 4, pp. 3447-3456, 2018.

[22] Z. Zhao, W. Zhou, D. Deng, J. Xia, and L. Fan, "Intelligent mobile edge computing with pricing in internet of things," IEEE Access, vol. 8, pp. 37727-37735, 2020.

[23] L. Fan, J. Xia, N. Yang, T. Q. Duong, G. Karagiannidis, and A. Nallanathan, "Opportunistic access point selection for mobile edge computing networks," IEEE Transactions on Wireless Communications, no. 99, 2020.

[24] Z. Junhui, Y. Tao, G. Yi, W. Jiao, and F. Lei, "Power control algorithm of cognitive radio based on non-cooperative game theory," China Communications, vol. 10, no. 11, pp. 143154, 2013.

[25] N. Zhao, X. Liu, F. R. Yu, M. Li, and V. C. M. Leung, "Communications, caching, and computing oriented small cell networks with interference alignment," IEEE Communications Magazine, vol. 54, no. 9, pp. 29-35, 2016.

[26] Z. Na, J. Wang, C. Liu, M. Guan, and Z. Gao, "Join trajectory optimization and communication design for UAV-enabled OFDM networks," Ad Hoc Networks, vol. 98, article 102031, 2020.

[27] Z. Na, J. Lv, F. Jiang, M. Xiong, and N. Zhao, "Joint subcarrier and subsymbol allocation-based simultaneous wireless information and power transfer for multiuser GFDM in iot," IEEE Internet of Things Journal, vol. 6, no. 4, pp. 5999-6006, 2019.

[28] Z. Na, J. Lv, M. Zhang, B. Peng, M. Xiong, and M. Guan, "GFDM based wireless powered communication for cooperative relay system," IEEE Access, vol. 7, pp. 50971-50979, 2019.

[29] Z. Na, Y. Wang, X. Li et al., "Subcarrier allocation based simultaneous wireless information and power transfer algorithm in 5G cooperative OFDM communication systems," Physical Communication, vol. 29, pp. 164-170, 2018.

[30] S. Lai, J. Xia, D. Zou, and L. Fan, "Intelligent secure communication for cognitive networks with multiple primary transmit power," IEEE Access, vol. 8, pp. 37343-37351, 2020.

[31] R. Zhao, "Deep reinforcement learning based mobile edge computing for intelligent internet of things," IEEE Access, no. 99, 2020.

[32] J. Xia, D. Deng, and D. Fan, “A note on implementation methodologies of deep learning-based signal detection for conventional MIMO transmitters," IEEE Transactions on Broadcasting, no. 99, pp. 1-2, 2020.

[33] K. He, "Ultra-reliable MU-MIMO detector based on deep learning for 5G/B5G-enabled IoT," EURASIP Journal on Wireless Communications and Networking, no. 99, 8 pages, 2020.

[34] J. Liao, J. Zhao, F. Gao, and G. Y. Li, "A model-driven deep learning method for massive MIMO detection," IEEE Communications Letters, no. 99, 2020.

[35] N. Zhao, Y. Cao, F. R. Yu, Y. Chen, M. Jin, and V. C. M. Leung, "Artificial noise assisted secure interference networks with wireless power transfer," IEEE Transactions on Vehicular Technology, vol. 67, no. 2, pp. 1087-1098, 2018.

[36] D. Deng, L. Fan, R. Zhao, and R. Q. Hu, "Secure communications in multiple amplify-and-forward relay networks with outdated channel state information," Transactions on Emerging Telecommunications Technologies, vol. 27, no. 4, pp. 494503, 2016.

[37] I. S. Gradshteyn and I. M. Ryzhik, Table of Integrals, Series, and Products, Academic Press, Elsevier Inc, San Diego, CA, USA, 7th edition, 2007.

[38] J. Xia, K. He, W. Xu, S. Zhang, L. Fan, and G. K. Karagiannidis, "A MIMO detector with deep learning in the presence of correlated interference," IEEE Transactions on Vehicular Technology, vol. 69, no. 4, pp. 4492-4497, 2020.

[39] G. Liu, Y. Xu, Z. He, Y. Rao, J. Xia, and L. Fan, “Deep learningbased channel prediction for edge computing networks toward intelligent connected vehicles," IEEE Access, vol. 7, pp. 114487-114495, 2019.

[40] K. He, Z. Wang, W. Huang, D. Deng, J. Xia, and L. Fan, "Generic deep learning-based linear detectors for MIMO systems over correlated noise environments," IEEE Access, vol. 8, pp. 29922-29929, 2020.

[41] Z. Zhao, R. Zhao, J. Xia et al., "A novel framework of threehierarchical offloading optimization for MEC in industrial IoT networks," IEEE Transactions on Industrial Informatics, vol. 16, no. 8, pp. 5424-5434, 2020.

[42] J. Xia, Y. Xu, D. Deng, Q. Zhou, and L. Fan, "Intelligent secure communication for internet of things with statistical channel state information of attacker," IEEE Access, vol. 7, pp. 144481-144488, 2019.

[43] C. Fan and Y. Ding, "Cooling load prediction and optimal operation of HVAC systems using a multiple nonlinear regression model," Energy and Buildings, vol. 197, pp. 7-17, 2019.

[44] C. Fan, Y. Ding, and Y. Liao, "Analysis of hourly cooling load prediction accuracy with data-mining approaches on different training time scales," Sustainable Cities and Society, vol. 51, article 101717, 2019. 